\title{
The Perception of Jordanian Population Towards Concomitant Administration of Food, Beverages and Herbs with Drugs and Their Possible Interactions: A Cross Sectional Study
}

\author{
Samar Thiab ${ }^{1}$, Muna Barakat ${ }^{1}$, Raja'a Al-Qudah ${ }^{2}$, Rana Abutaima ${ }^{3}$, Rahma Jamal ${ }^{1}$, and \\ Philip Riby ${ }^{4}$ \\ ${ }^{1}$ Applied Science Private University \\ ${ }^{2}$ Applied Science Private University Faculty of Pharmacy \\ ${ }^{3}$ Zarqa Private University \\ ${ }^{4}$ The University of Manchester
}

August 6, 2020

\begin{abstract}
Background Many people are used to administering their drugs with food, beverages, or herbs, which may contain chemicals that interfere with the prescribed drugs that could potentially lead to changes in their efficacy or safety and alteration in their pharmacokinetic properties. Objective To assess the extent of perception and use of food, beverages and herbs alongside with conventional drugs and their potential interactions among Jordanian society. Methods This descriptive cross-sectional survey was conducted in Jordan (20 April - 5 May 2020). The survey was developed using Google forms, validated and distributed via social media platforms. Data was analyzed using Statistical Package for Social Sciences-24. Main outcome measure Use and perception of food, beverages, herbs and their drug interactions among Jordanians. Results Of all participants (n $=789$ ), $77.8 \%$ were females, $46.2 \%$ were 50 -year-old, $69.7 \%$ were married, $70.8 \%$ were medically insured, and $51.1 \%$ had a bachelor's degrees. Seventy percent of the study participants reported use of medicinal plants. About $66 \%$ of participants agreed that medicinal plants or herbs could treat diseases and $58.6 \%$ thought that medications could interact with drugs. In general, the participants' knowledge about food/beverage/herb-drug interactions was considered poor. However, linear regression analysis illustrated that the level of knowledge was significantly affected (p-value $<0.05$ ) by gender, marital status, social status, the educational level, and employment sector. Conclusion Jordanians have a positive perception towards herbs and their ability to treat diseases. However, their knowledge about food/beverage-drug interactions was poor. This call needs to enhance the community awareness on food/beverage/herb-drug interactions.
\end{abstract}

\section{What does this article add?}

- Emphasizes the need to provide training for Jordanian community pharmacists to enhance their patient counseling skills and to focus on common food/beverage/herb-drug interactions while serving patients.

- Emphasizes the need to provide Continuing Professional Development (CPD) sessions for healthcare providers (i.e. physicians, nurses, pharmacists ...etc.) on building rapport with patients and highlighting important food/beverage/herb-drug interactions.

\section{Introduction}

According to the US-Food and Drug Administration (FDA), the interaction between drugs and food or beverages can mainly affect the activity of drugs, either by increasing or decreasing their medicinal effects, causing unexpected side effects, or leading to the development of a new pharmacological effects that drugs would not produce originally (1-3). 
Many people all over the world are used to taking their drugs with food, beverages, or herbs (4). For example, approximately, one in five Asian people take their medications concurrently with herbal products and juices (4). These products may contain chemicals that interfere with the prescribed drugs leading to changes in their bioavailability and alteration of their pharmacokinetic properties (5). People usually take their drugs with herbs or beverages such as juices seeking for health benefits or to taste-mask the bitterness of some drugs $(6)$.

Some plants or herbs are well known for their ability to interact with drugs (5). The most common example is St. John's wort (Hypericum perforatum ), which is used for mild depression and known for its interactions with various drugs (7). Examples of these drugs include antidepressants like serotonin reuptake inhibitors, immunosuppressants such as ciclosporin and tacrolimus, human immunodeficiency virus( HIV) medications, and hormonal contraceptives (2,7). Ginkgo (Ginkgo biloba), usually used to improve memory and blood circulation, can inhibit platelet-activating factor; thus, it is well-known for its interaction with warfarin resulting in an increased risk of bleeding $(2,8)$. In addition, since the discovery of grapefruit juice interaction with felodipine; an antihypertensive medication, many studies were conducted on other juices to investigate their tendency to perpetrate potential food-drug interaction (9-11).

Numerous patients do not mention the concurrent administration of their medications with food, beverages, or herbs to their physicians or pharmacists due to many reasons (5). Firstly, physicians do not usually ask their patients about their method of drug administration and the intake of other health products such as herbs or over-the-counter (OTC) drugs, as they usually focus on the patients' conventional drugs (12). Secondly, physicians do not have enough knowledge about herbal remedies uses, side effects and drug interactions (1315). Thirdly, either patients or their healthcare providers, do not consider natural products an important thing to mention (8). Finally, patients fear the potential disapproval of their healthcare providers regarding their use of non-prescribed drugs and herbs $(16,17)$. As a result, patients have fewer opportunities to learn about potential interactions with their drugs or other herbal products $(5,18)$.

Several studies were conducted to assess the awareness of healthcare professionals about simultaneous intake of food, beverages and herbal products with drugs $(19,20)$, However, there are a limited number of studies investigating the awareness of the public community toward such practice $(21,22)$, which can have serious health repercussions on individuals (23-25).

This is the first study assessing the extent of perception and use of food, beverages, and herbs alongside conventional drugs among Jordanian society.

\section{Materials and Methods}

Study design and participants

The study objectives were addressed in a descriptive cross-sectional electronic survey. This study was conducted in Jordan from 20 April to 5 May 2020. The survey was developed and validated to solicit anonymous responses, which were treated confidentially. Eligible participants were any interested Jordanian citizens living in Jordan. The participants in our study were recruited through social media platforms: Facebook, WhatsApp, LinkedIn, Twitter.

Participation in the study was voluntary and did not pose any risk to participants. Potential participants who completed the survey were considered to have given informed consent for participation in the study.

\section{Ethics Approval}

Ethical approval for the study was obtained from the Faculty of Pharmacy, Applied Science Private University (Approval number: 2020-PHA-4).

Survey development, validation, and reliability

The survey was developed after reviewing validated surveys in literature $(1,15,21,22,26)$ and was designed using the general principles of good survey design (27). Several sources were used to generate a pool of 
questions considered to be relevant to the study objectives $(1,13,26,28)$. The online survey was created using the technology of Google Forms provided by Google and was constructed in English, but it was delivered to participants in Arabic, the formal language in Jordan. The survey contained multiple-choice questions and could be completed within 15 minutes.

To ensure face validity, the first draft of the survey was evaluated by twelve independent academics who have previous experience in food/beverage/herb-drug interactions and a statistician. The provided comments and feedback were considered and incorporated where appropriate to develop the final version of the survey. The survey was then translated from English into Arabic and back by two senior academic staff members who are fluent in both languages. The questions were free from medical jargon or difficult terminology and popular trade names were mentioned to help participants recognize the drugs of interest. Finally, the survey was piloted on a sample of 25 academics and 25 non-academic people to enhance clarity, readability, understandability, and confirm its applicability to the Jordanian population. Internal consistency reliability was tested by the Cronbach's alpha coefficient.

The final version of the survey contained four parts with the first part (Part A) comprised of nine questions about sociodemographic information; the second part (Part B) consisted of six questions about the perception of the community toward food/beverage/herb-drug interactions; the third part (Part C) consisted of five questions, was designed to assess the attitude and practice of community toward the food/beverage/herbdrug interactions; the fourth part (Part D) included five questions to evaluate the knowledge about common food/beverage/herb-drug interactions. The last part was used to calculate the knowledge score (out of 5), depending on the participants' correct answers.

Sample size

Currently, the number of Jordanian citizens living in Jordan is 10.554 million (29). Based on that, calculating the sample size using a margin of error of $5 \%$, confidence level of $95 \%$, and response distribution of $50 \%$, a minimum sample size of 385 is needed (30). The number of participants in this study was 789 .

Statistical analyses

Completed surveys were extracted from Google Forms as an Excel sheet and were then exported to Statistical Package for Social Sciences version 24.0 (SPSS Inc., Chicago, IL, USA) for the statistical analysis. Descriptive statistics including percentages; means and frequency distribution were calculated for each question. Descriptive and univariate correlation analyses with the Pearson correlation coefficient (r) were used for the correlation at the $5 \%$ significance level. A $p$-value of $<0.05$ represents a significant difference. Factors affecting knowledge score about food/beverage/herb-drug interactions, were screened using simple and multiple linear regression.

\section{Results}

Demographic characteristics

A total of 798 surveys were received, and 9 were excluded due to incompleteness. Accordingly, 789 responses were included in the study analysis. The sociodemographic characteristics of the participants are represented in Table 1. There was a predominance of female participants $614(77.8 \%)$ compared with males. Most of the participants $368(46.2 \%)$ were 40-65-year-old in age, married 550 (69.7\%), medically insured 559 (70.8\%), and had bachelor's degrees $408(51.7 \%)$. One-third of them were employees in a non-health sector 159 $(20.2 \%)$ and $192(24.3 \%)$ were housewives. Most of the participants $585(74.1 \%)$ were living in the city and concentrated in Amman, the capital of Jordan, where $42 \%$ of Jordan's population reside (29).

Perception of the community towards food/beverage/herb use concurrently with drugs and their possible interactions

The perception of the community participants towards the use of food, beverages and herbs concurrently with drugs and their possible interaction is represented in Table 2, Section 1. The study findings showed that $524(66.4 \%)$ of the participants agreed that food and herbs could treat diseases. More than half of them 
$462(58.6 \%)$ thought that medications could interact with the action of drugs, change their action or cause side effects with food, beverages, and herbs. About $34 \%$ of the responses disputed that the concomitant use of drugs with medicinal plants or herbs would improve their effects, while almost similar percentage of participants (38.4\%) answered 'sometimes'. Similar findings were found upon asking if treating diseases with medicinal plants or herbs is better than conventional drugs, as most of them answered 'no' and 'sometimes, $36.9 \%$ and $45.6 \%$, respectively. Finally, a total of $473(59.9 \%)$ participants reported that children and the elderly are the most likely categories to experience drug interactions with food, beverages, and herbs.

Attitude and practice of community towards the use of food/beverage/herb concurrently with drugs

During the past twelve months, only $41(5.2 \%)$ participants have used drugs concomitantly with medicinal plants or herbs on daily basis, while the other participants use that combination less frequently. Furthermore, $641(81.2 \%)$ of the study participants admitted that they do not administer their drugs with any drinks other than water, while the others $(18.8 \%)$ said that the medication was consumed with juices, tea, coffee, or milk (Table 2, Section 2).

Seventy percent of the study participants reported that they used medicinal plants or herbs to treat specific diseases or to boost their immune system. Then, they mentioned several medicinal plants and herbs they used frequently. A short answer question was used to identify these herbs/plants as illustrated in Figure 1, A. Upon asking the participants about the potentially treatable diseases using medicinal plants and herbs, they were able to choose more than one option (respiratory, gastrointestinal track. . etc.). A total of 305 (38.7\%) chose multiple options, mostly respiratory-related illnesses and gastrointestinal tract 'stomach/intestinal problems. About 166 (21\%) of the participants selected the respiratory illnesses 'cold, cough and influenza' alone. A similar percentage of the participants, 165 (21\%) preferred not to use medicinal plants and herbs to treat diseases, as can be seen in Figure 1, B.

Knowledge about common food/beverage/herb-drug interactions

Usually, knowledge about anything should have a source. With regards to the sources of information about herbal products, study participants reported that they obtain their information mostly from the internet which was the main resource of information 212 (26.6\%), followed by physicians 74 (9.3\%), (Figure 2). Half of the study participants $427(53.6 \%)$ selected more than one answer including internet, physicians, family, and pharmacists as seen in Figure 2.

On the other hand, the participants' knowledge about some commonly used drugs that has the potential to interact with some food/beverage/herb was examined and scored out of five (the total number of questions), the results are demonstrated in Table 3 . The overall knowledge score was poor $0.99 \pm 1.22$ (mean $\pm \mathrm{SD}$, out of 5). About one-third of the study participants, 165 (33.6\%) correctly answered the fact that they should avoid taking tetracycline antibiotics with milk. Only, 60 (7.6\%) participants had known that the consumption of excessive amounts of cranberry juice should be avoided when they are on the anticoagulant warfarin.

Multiple linear regression analysis of factors affecting participants' knowledge scores highlighted that the female gender, unmarried participants, university degree holders, and health sector employees were significantly $(p$-value $<0.05)$ affecting the participants' knowledge score (Table 4$)$.

\section{Discussion}

This is the first national study to assess the extent of knowledge about food/beverage/-drug interactions among Jordanian citizens. Drug interactions are one of the major drug-related problems, but usually neglected due to the lack of sufficient knowledge and public awareness $(21,31)$.

This study revealed that the majority of participants acknowledged the importance of medicinal plants and herbs in treating diseases. This shows an agreement to the findings of similar studies conducted in Morocco (32), Tanzania (33), the United Kingdom (17), and Surinamese migrants in the Netherlands (34). Many scientific studies support the use of plants to treat diseases under medical supervision (35). For example, Aloe vera is useful for wound healing and recovery from burn injury (36), Clove oil (Eugenia caryophyllus ) 
is used for the relief of toothache (37), and ginger (Zingiber officinale) is well-known as a reliever of morning sickness and motion sickness (38).

The results of this study showed that participants used medicinal plants and herbs to treat diseases, mainly respiratory tract illnesses or to boost their immunity. Similar findings by Zaffani et al, showed that Italian women used medicinal plants and herbs to boost their immune system and to cure respiratory disorders (39). In addition, our study participants mentioned several plants and herbs that they usually used, including mint (Mentha piperita), melissa (Melissa officinalis), garden cress (Lepidium sativum), cumin (Cuminum cyminum ), fenugreek (Trigonella foenum-graecum), mugworts (Artemisia vulgaris ), fennel (Foeniculum vulgare) and turmeric (Curcuma longa). Those plants are commonly well-known in the Middle East and Jordan, readily available and affordable $(40,41)$.

One of the most concerning findings of this study was related to the poor level of knowledge among participants regarding some food/beverage/herb-drug interactions. Although the majority of participants in this survey were from the capital Amman and more than half of them had a university degree, their knowledge regarding food/beverage-drug interactions was poor. This also it indicates that the information leaflet provided with drugs is often not read. The knowledge score was calculated for five common drug classes in Jordan (42). The first was about the consumption of excessive amounts of chocolate with antidepressants, particularly monoamine oxidase inhibitors (MAOIs) due to their caffeine content, which could enhance the hypertensive effect of MAOIs $(43,44)$. The second was about the interaction of tetracycline antibiotics with milk, which is one of the most common interactions that lead to a significant impairment of the antibiotic's absorption causing a decrease in its serum concentration (45-47). The third was about the need to avoid the consumption of excessive amounts of bananas when taking angiotensin-converting enzyme (ACE) inhibitors antihypertensives which may increase the risk of developing hyperkalemia, due to its high potassium content. Hyperkalemia can cause irregular heartbeat and heart palpitation (48-50). The fourth was about the need to avoid excessive intake of cranberry and cranberry juice concurrently with vitamin $\mathrm{K}$ antagonists anticoagulants, such as warfarin, as it may increase the international normalized ratio (INR) and increase the risk of bleeding (51-54). The last point was related to grapefruit, which is known for its numerous interactions with many drugs and sometimes can lead to fatal complications (55-58). The results showing a poor knowledge about food/beverage/herb-drug interactions among participants are consistent with the findings of a study conducted in the United States of America, where it was found that only one-third of medicinal herb users had ever discussed that with their healthcare providers, which leaves the majority without sufficient information (15). The same was also found in a study conducted in the United Kingdom where the women who participated were found to have little knowledge about herb-drug interactions as they rarely disclosed their use of herbs and other medicinal plants to their healthcare providers as well (17).

The lack of sufficient knowledge among Jordanians, emphasizes the need for extensive educational courses, workshops, and continuous awareness programs to highlight the risk of concurrent intake of food, beverages and herbs with drugs, primarily for healthcare providers. Hence, our Jordanian community relay comprehensively on the advice provided by healthcare providers, which would be reflected by their knowledge, awareness, and appropriate use of drugs, as shown by several published studies $(17,20,39)$. Interestingly, this aspect was also requested by several healthcare professionals as reported in a study conducted in Lebanon in which pharmacists were interested in getting courses and workshops to help them better understand possible drug interactions (59). As a result, there is a need for proper patient information gathering by community pharmacists, not only by their physicians to help provide better

Many factors were affecting the knowledge score among the study participants, including the education level, as university graduates and postgraduates had higher knowledge levels in comparison with participants who had only a high school certificate or a diploma, which is consistent with other studies (20) In one study conducted in India it was found that professors had good knowledge about food-drug (21). interactions compared to other participants because they had better expertise (20). Moreover, workers in the healthcare sectors had higher knowledge than participants who had other types of jobs or those who are jobless, which is also consistent with studies in the literature (21). Sajid et al . found that clinical pharmacologists had 
more awareness of food-drug interaction compared to doctors and nurses, and non-medically qualified people had even less awareness (21). On the other hand, unmarried participants had higher knowledge than other participants, which was an interesting finding for the researchers because it was expected that married participants would have better knowledge, particularly if they have children and went through pregnancy, which needs extra care regarding drug interactions (60-62).

In general, Jordanians are aware of the existence of food/beverage/herb-drug interactions. However, they have little knowledge about what food beverages or herbs interact with common medications. This highlights the need to encourage patients to discuss their use of natural products with their healthcare providers, who also need proper education about this matter.

Limitations

The first limitation of this study was patient self-selection because this survey was conducted online due to the novel coronavirus pandemic and the public quarantine currently imposed in Jordan, so only people who use the Internet and social media were able to participate.

The second limitation is the representativeness of the sample to the population of Jordan. Females actively participated in this survey are more than males. Most of the participants were from the capital Amman, which may not be generalizable to the rest of the cities in the county. However, this provides an opportunity for future studies to access the perception, attitude, and practice regarding drug interactions and more extensively nationwide.

\section{Conclusion}

Generally, Jordanians have a positive perception towards herbs and their ability to treat diseases. However, their knowledge about food/beverage-drug interactions was poor. This calls attention for the need to develop proper educational programs to enhance community awareness and knowledge about food/beverage/herbdrug interactions to help avoid complications or side effects associated with such types of interaction. This can be done in many ways including awareness campaigns sponsored by pharmaceutical companies, hospitals, pharmacies, or the Ministry of Health. In addition to the distribution of pamphlets or leaflets regarding this matter or even hosting healthcare providers who are well-educated about food/beverage/herb-drug interactions in national television and interviewing them by local newspapers to help educate people.

\section{Funding}

This study was funded by the Deanship of Academic Research at Applied Science Private University, Amman, Jordan.

\section{Conflict of interests}

The authors have no conflict of interest to declare.

\section{References}

1. Owens C, Toone T. A Survey of Dietary Supplement Knowledge, Attitudes, and Use in a Rural Population. J Nutr Food Sci. 2014;4(5).

2. Fugh-Berman A. Herb-drug interactions. Lancet. 2000;355(9198):134-8.

3. FDA. Drug Interactions: What You Should Know: U.S. Food and Drug Administration; 2013 [Available from: https://www.fda.gov/drugs/resources-you-drugs/drug-interactions-what-you-should-know.

4. Marcus DM, Grollman AP. Botanical medicines-the need for new regulations. N Engl J Med. 2002;347(25):2073-20176.

5. Henriksen K, Battles JB, Keyes MA, Grady ML. Risk of concurrent use of prescription drugs with herbal and dietary supplements in ambulatory care. Advances in Patient Safety: New Directions and Alternative Approaches. 2008;4(Technology and Medication Safety). 
6. Hanley MJ, Cancalon P, Widmer WW, Greenblatt DJ. The effect of grapefruit juice on drug disposition. Expert Opini Drug Met 2011;7(3):267-86.

7. Mannel M. Drug interactions with St John's wort. Drug Safety. 2004;27(11):773-97.

8. Skinner CM. Preoperative use of herbal medicines: a patient survey. Br J Anaesth. 2002;89(5):792-5.

9. Mouly S, Lloret-Linares C, Sellier PO, Sene D, Bergmann JF. Is the clinical relevance of drug-food and drug-herb interactions limited to grapefruit juice and Saint-John's Wort? Pharmacol Res. 2017;118:82-92.

10. Mutalik M, Sanghavi D. Review of drug interactions: A comprehensive update. J Pharm Res Int. 2014;4(8):954-80.

11. Chen M, Zhou SY, Fabriaga E, Zhang PH, Zhou Q. Food-drug interactions precipitated by fruit juices other than grapefruit juice: An update review. J Food Drug Anal. 2018;26(2S):S61-S71.

12. Wynia MK, Eisenberg DM, Wilson IB. Physician-patient communication about complementary and alternative medical therapies: a survey of physicians caring for patients with human immunodeficiency virus infection. J Altern Complement Med 1999;5(5):447-56.

13. Giveon SM, Liberman N, Klang S, Kahan E. A survey of primary care physicians' perceptions of their patients' use of complementary medicine. Complement Ther Med. 2003;11(4):254-60.

14. Winslow LC, Shapiro H. Physicians want education about complementary and alternative medicine to enhance communication with their patients. Arch Intern Med 2002;162(10):1176-81.

15. Wheaton AG, Blanck HM, Gizlice Z, Reyes M. Medicinal herb use in a population-based survey of adults: prevalence and frequency of use, reasons for use, and use among their children. Ann Epidemiol. $2005 ; 15(9): 678-85$.

16. Tasaki K, Maskarinec G, Shumay DM, Tatsumura Y, Kakai H. Communication between physicians and cancer patients about complementary and alternative medicine: exploring patients' perspectives. Psychooncology. 2002;11(3):212-20.

17. Vickers KA, Jolly KB, Greenfield SM. Herbal medicine: women's views, knowledge and interaction with doctors: a qualitative study. BMC Complement Altern Med. 2006;6:40.

18. Eisenberg DM, Davis RB, Ettner SL, Appel S, Wilkey S, Van Rompay M, et al. Trends in alternative medicine use in the united states. Trends in alternative medicine use in the United States, 1990-1997: results of a follow-up national survey. 1998;280(18):1569-75.

19. Ndosi ME, Newell R. Nurses' knowledge of pharmacology behind drugs they commonly administer. J Clin Nurs. 2009;18(4):570-80; discussion 620.

20. Benni JM, Jayanthi MK, Tubaki BR, Renuka M. Knowledge and awareness of food and drug interactions (FDI): a survey among health care professionals. International Journal of Pharmacology and Clinical Sciences. 2012;1(4):97-105.

21. Sajid S, Sultana R, Masaratunnisa M, Naaz S, Adil M. A questionnaire study of food - drug interactions to assess knowledge of people from diverse backgrounds. Asian J Med Health. 2017;5(2):1-9.

22. Kuo GM, Hawley ST, Weiss LT, Balkrishnan R, Volk RJ. Factors associated with herbal use among urban multiethnic primary care patients: a cross-sectional survey. BMC Complement Altern Med. 2004;4:18.

23. Genser D. Food and drug interaction: consequences for the nutrition/health status. Ann Nutr Metab. 2008;52 Suppl 1:29-32.

24. Sørensen JM. Herb-drug, food-drug, nutrient-drug, and drug-drug interactions: mechanisms involved and their medical implications. J Altern Complement Med. 2002;8(3):293-308.

25. Schmidt LE, Dalhoff K. Food Drug Interactions. Drugs. 2002;62(10):1481-502. 
26. Singh JA, Bharat A, Edwards NL. An internet survey of common treatments used by patients with gout including cherry extract and juice and other dietary supplements. J Clin Rheumatol. 2015;21(4):225-6.

27. Boynton PM, Greenhalgh T. Selecting, designing, and developing your questionnaire. BMJ. 2004;328(7451):1312-5.

28. Marinac JS, Buchinger CL, Godfrey LA, Wooten JM, Sun C, Willsie SK. Herbal products and dietary supplements: a survey of use, attitudes, and knowledge among older adults. J Am Osteopath Assoc. 2007;107(1):13-23.

29. Department of Statistics. Population Estimates in Jordan 2019 [Available from: http://dosweb.dos.gov.jo/DataBank/Population_Estimares/PopulationEstimates.pdf.

30. Taherdoost H. Determining sample size; how to calculate survey sample size. Int J Econs \& Mgmt. 2017;2:237-9.

31. Asahina Y, Hori S, Sawada Y. Community pharmacists' attitudes relating to patients' use of health products in Japan. Int J Clin Pharm. 2012;34(4):529-37.

32. Eddouks M, Maghrani M, Lemhadri A, Ouahidi ML, Jouad H. Ethnopharmacological survey of medicinal plants used for the treatment of diabetes mellitus, hypertension and cardiac diseases in the south-east region of Morocco (Tafilalet). J Ethnopharmacol. 2002;82(2-3):97-103.

33. Maregesi SM, Ngassapa OD, Pieters L, Vlietinck AJ. Ethnopharmacological survey of the Bunda district, Tanzania: plants used to treat infectious diseases. J Ethnopharmacol. 2007;113(3):457-70.

34. van Andel T, Westers P. Why Surinamese migrants in the Netherlands continue to use medicinal herbs from their home country. J Ethnopharmacol. 2010;127(3):694-701.

35. Kaur J, Kaur S, Mahajan A. Herbal medicines: possible risks and benefits. American Journal of Phytomedicine and Clinical Therapeutics. 2013;2:226-39.

36. Sahu PK, Giri DD, Singh R, Pandey P, Gupta S, Shrivastava AK, et al. Therapeutic and medicinal uses of Aloe vera: a review J Pharm Pharmacol. 2013;04(08):599-610.

37. Cortés-Rojas DF, de Souza CRF, Oliveira WP. Clove (Syzygium aromaticum): a precious spice. Asian Pac J Trop Biomed. 2014;4(2):90-6.

38. Lien HC, Sun WM, Chen YH, Kim H, Hasler W, Owyang C. Effects of ginger on motion sickness and gastric slow-wave dysrhythmias induced by circular vection. Am J Physiol Gastrointest Liver Physiol 2003;284(3):G481-G9.

39. Zaffani S, Cuzzolin L, Benoni G. Herbal products: behaviors and beliefs among Italian women. Pharmacoepidemiol Drug Saf. 2006;15(5):354-9.

40. Afifi FU, Abu-Irmaileh B. Herbal medicine in Jordan with special emphasis on less commonly used medicinal herbs. J Ethnopharmacol. 2000;72(1-2):101-10.

41. Abdelhalim A, Aburjai T, Hanrahan J, Abdel-Halim H. Medicinal plants used by traditional healers in Jordan, the Tafila region. Pharmacogn Mag. 2017;13(Suppl 1):S95-S101.

42. JFDA. Jordan National Drug Formulary: Jordan Food and Drug Administration; 2011 [Available from: http://www.jfda.jo/EchoBusV3.0/SystemAssets/PDF/AR/JNDF-Book/JNDF\%20V2\%202011.pdf.

43. van der Hoeven N, Visser I, Schene A, van den Born BJ. Severe hypertension related to caffeinated coffee and tranylcypromine: a case report. Ann Intern Med. 2014;160(9):657-8.

44. van den Berg D, Zoellner KR, Ogunrombi MO, Malan SF, Terre'Blanche G, Castagnoli N, Jr., et al. Inhibition of monoamine oxidase B by selected benzimidazole and caffeine analogues. Bioorg Med Chem. 2007;15(11):3692-702. 
45. Neuvonen PJ. Interactions with the absorption of tetracyclines. Drugs. 1976;11(1):45-54.

46. Leyden JJ. Absorption of minocycline hydrochloride and tetracycline hydrochloride: effect of food, milk, and iron. J Am Acad Dermatol. 1985;12(2):308-12.

47. Jung H, Peregrina AA, Rodriguez JM, Moreno-Esparza R. The influence of coffee with milk and tea with milk on the bioavailability of tetracycline. Biopharm Drug Dispos. 1997;18(5):459-63.

48. Opie LH. Drug interactions of antihypertensive agents. S Afr Fam Pract. 2014;54(2):S23-S5.

49. Burnakis TG, Mioduch HJ. Combined therapy with captopril and potassium supplementation. A potential for hyperkalemia. Arch Intern Med. 1984;144(12):2371-2.

50. Shionoiri H. Pharmacokinetic drug interactions with ACE inhibitors. Clin Pharmacokinet. 1993;25(1):20-58.

51. Hamann GL, Campbell JD, George CM. Warfarin-cranberry juice interaction. Ann Pharmacother. 2011;45(3):e17.

52. Grant P. Warfarin and cranberry juice: an interaction? J Heart Valve Dis. 2004;13(1):25-6.

53. Suvarna R, Pirmohamed M, Henderson L. Possible interaction between warfarin and cranberry juice. BMJ. 2003;327(7429):1454.

54. Rindone JP, Murphy TW. Warfarin-cranberry juice interaction resulting in profound hypoprothrombinemia and bleeding. Am J Ther. 2006;13(3):283-4.

55. Seden K, Dickinson L, Khoo S, Back D. Grapefruit-drug interactions. Drugs. 2010;70(18):2373-407.

56. Mertens-Talcott SU, Zadezensky I, De Castro WV, Derendorf H, Butterweck V. Grapefruit-drug interactions: can interactions with drugs be avoided? J Clin Pharmacol. 2006;46(12):1390-416.

57. Bailey DG, Dresser G, Arnold JMO. Grapefruit-medication interactions: Forbidden fruit or avoidable consequences? Can Med Assoc J. 2013;185(4):309-16.

58. Stump AL, Mayo T, Blum A. Management of grapefruit-drug interactions. Am Fam Physician. 2006;74(4):605-8.

59. Hijazi MA, Shatila H, El-Lakany A, Aboul Ela M, Kharroubi S, Alameddine M, et al. Beliefs, practices and knowledge of community pharmacists regarding complementary and alternative medicine: national cross-sectional study in Lebanon. BMJ Open. 2019;9(3):e025074.

60. Faqi AS, Holm KA. Metabolism and Drug-Drug Interaction in Pregnant Mother/Placenta/Fetus. Dev Reprod Toxicol. Methods in Pharmacology and Toxicology2016. p. 1-16.

61. Ferracini AC, Rodrigues AT, Visacri MB, Stahlschmidt R, Silva N, Surita FG, et al. Potential Drug Interactions and Drug Risk during Pregnancy and Breastfeeding: An Observational Study in a Women's Health Intensive Care Unit. Rev Bras Ginecol Obstet. 2017;39(6):258-64.

62. Rubinchik-Stern M, Eyal S. Drug Interactions at the Human Placenta: What is the Evidence? Front Pharmacol. 2012;3:126.

\begin{tabular}{ll}
\hline Table 1. Sociodemographic characteristics $(\mathbf{N}=\mathbf{7 8 9})$ & $\begin{array}{l}\text { Table 1. Sociodemographic characteristics }(\mathbf{N}=\mathbf{7 8 9}) \\
\text { Fariable }\end{array}$ \\
Gender & $614(77.8)$ \\
Female & $175(22.2)$ \\
Male & \\
Age & $13(1.6)$ \\
$<18$ years old & $81(10.3)$ \\
$18-25$ years old &
\end{tabular}




\section{Social status}

Single

$196(24.8)$

Married

$550(69.7)$

Divorced/ Widowed

$43(5.5)$

Education level

High school certificate

$76(9.6)$

Diploma

$140(17.7)$

Bachelor's degree

$408(51.7)$

postgraduate's degree

145 (18.4)

Others

$20(2.5)$

\section{Occupation}

University student

$46(5.8)$

Housewife

$192(24.3)$

Employee in the health sector

$156(19.8)$

$159(20.2)$

Employee in a non-health sector

$85(10.8)$

Academics

$71(9.0)$

Retired

$63(8.0)$

Freelancers or business owner (institution or company)

$16(2.0)$

Permanent place of residence

Village

$35(4.4)$

City

Badia

Location of work

The capital of Jordan (Amman)

$585(74.1)$

Middle of Jordan

$84(10.6)$

North of Jordan

$51(6.5)$

South of Jordan

$10(1.3)$

East of Jordan

$20(2.5)$

West of Jordan

$39(4.9)$

Monthly Income

$<250$ JOD

$83(10.5)$

250-500 JOD

$255(32.3)$

500-750 JOD

$140(17.7)$

$134(17.0)$

750-1000 JOD

$177(22.4)$

Health Insurance Status

Table2. Perception, attitude and practice of participants toward the use of drugs concurrently with food, beverages and herbs and their possible interactions $(\mathrm{N}=789)$

Question

In your opinion, could herbs treat diseases?

Yes

Yes - "only if my doctor tells me to"

No 
Neutral

In your opinion, can drugs interact (interfere with the action of drugs or cause side effects) with food, beve Yes

No

Sometimes

I am not sure

In your opinion, is using drugs herbs better than just using them?

Yes

No

Sometimes

I am not sure

In your opinion, is the treatment with herbs better than conventional drugs?

Yes

No

Sometimes

I am not sure

In your opinion, which is most likely to experience drug interactions with food, beverages or herbs?

Infants

Children

Adults

Elderly

Children and Elderly

Infants, Children and Elderly

Attitude and practice

How often have you used your drugs with herbs in the past twelve months?

Daily

Often

When I'm not feeling well

Sometimes

Never

Did you take your drugs with a drink other than water?

Yes, with juice

Yes, with tea

Yes, with coffee

Table . Evaluation of study about some foodbeverage-drug interactions $(\mathrm{N}=789)$.

Which of the following food should not be

Correct Answer N (\%)

administered in excessive amounts when you

are on antidepressants (monoamine oxidase

inhibitors)? Chocolate Ice Cream Fruit

Vegetables I don't Know There is nothing to avoid

Which of the following beverages should be

avoided when taking tetracycline (e.g.

Doxydar@, Doxymed(A, Unidox(B)

antibiotics? Milk Fruit Juices Tea Coffee I don’t

Know There is nothing to avoid

$106(13.4)$ 
Which of the following food should not be

$123(15.6)$ administered in excessive amounts when you are on any ACE inhibitor (e.g. Capocardß), Angiotec(B), Zestril@) an antihypertensive agent? Green leafy vegetables Banana Orange Apple I don't Know There is nothing to avoid Which of the following juices should not be administered in excessive amounts when you are on warfarin, a blood thinner (e.g.

Orfarin(B)? Pineapple Mango Cranberry Apple I don't Know There is nothing to avoid

Which of the following options could interact with many medications and can cause fatal side effects? Apple Orange Grapefruit Mango I don't Know There is nothing to avoid Total Knowledge Score (out of 5), Mean \pm SD

*The answers to the above questions are: 1. Chocolate, 2. Milk, 3. Banana, 4. Cranberry juice and 5. Grapefruit.

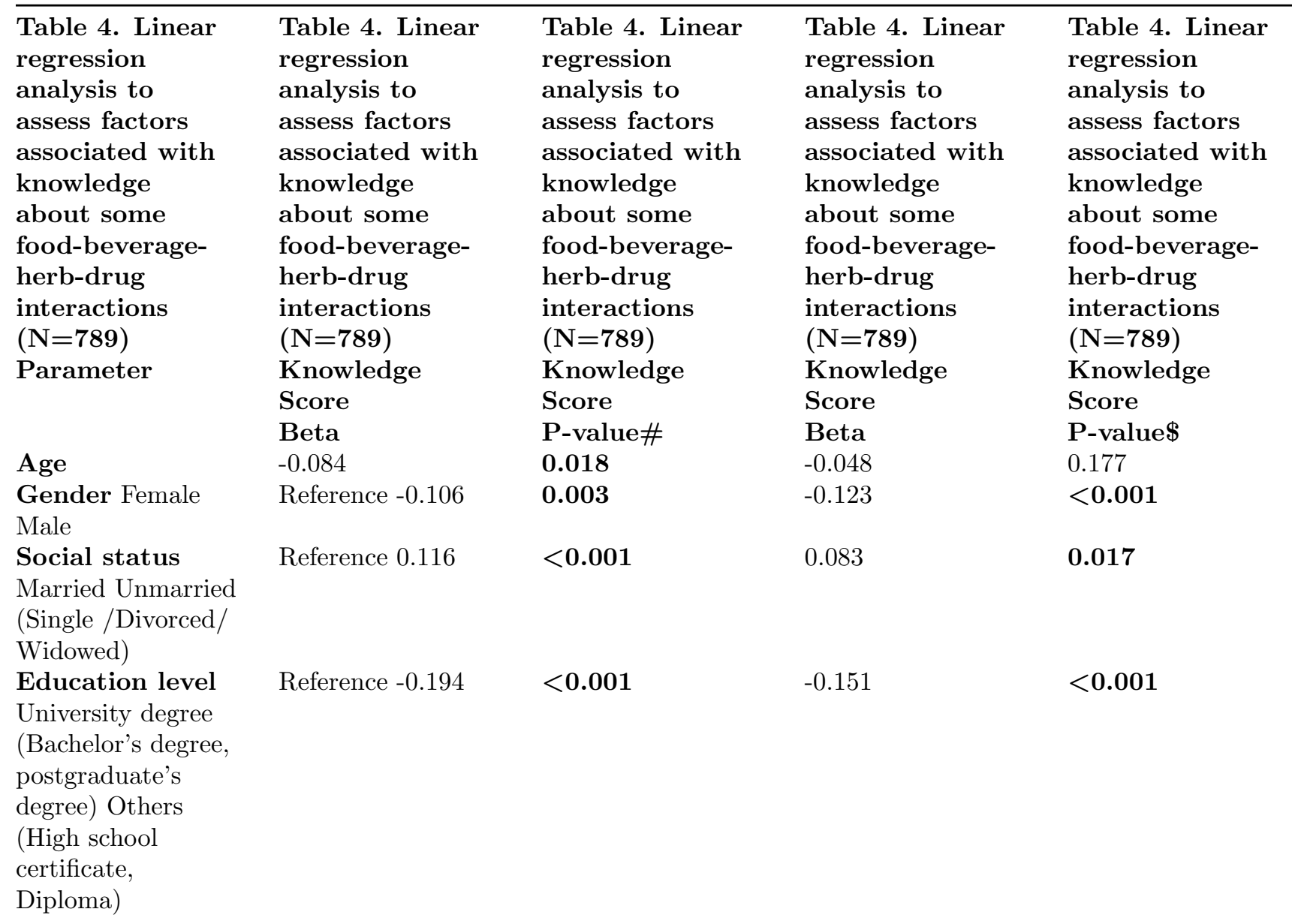


Occupation An

Reference -0.336

$<0.001$

$-0.308$

$<0.001$

employee in the

health sector Others

(Employee in a

non-health sector,

Academics, Retired,

Freelancers or

business owner,

University student,

Housewife)

Permanent place

of residence City

Badia and Village

Monthly income

$<500$ JOD $>500$

JOD

Health insurance

Reference -0.032

0.372

0.036

0.307

Reference 0.024

0.507

0.036

0.304

Reference -0.075

0.036

$-0.032$

0.338

status Insured

Uninsured

\# using simple linear regression, \$ using multiple linear regression, significant at 0.05 significance level (bolded numbers ).

\section{Figure legends}

Figure 1. A)The percentage of participants who used medicinal plants or herbs to treat specific diseases or boost their immune system. The participant who answered "Yes", addressed the plants/herbs they used to use in such case. B) The diseases that participants would treat using medicinal plants or herbs. $(\mathrm{N}=789)$.

Figure 2. Sources of information about herbs and herbal products among study participants $(\mathrm{N}=789)$.

(A

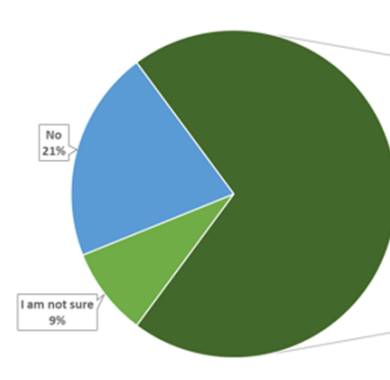

Figure 1. A)The percentage of participants who used medicinal plants or herbs to treat specific diseases or boost their immune system. The participant who answered "Yes", addressed the plants/herbs they used to use in such case. B) The diseases that participants would treat using medicinal plants or herbs. $(\mathrm{N}=789)$.
(B

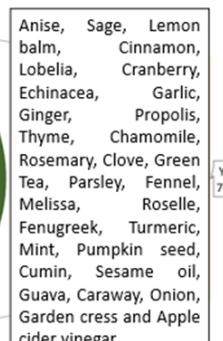

others $3.2 \%$

Mental and spiritual health $0.3 \%$

Headache/migraine $0.4 \%$

Arthritis/joint pains || 1.1\%

Menstrual/uterus problems $11.3 \%$

Sleep problems

Cold/influenza/cough
III.0\%

Respiratory and GIT illnesses

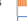

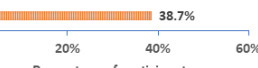




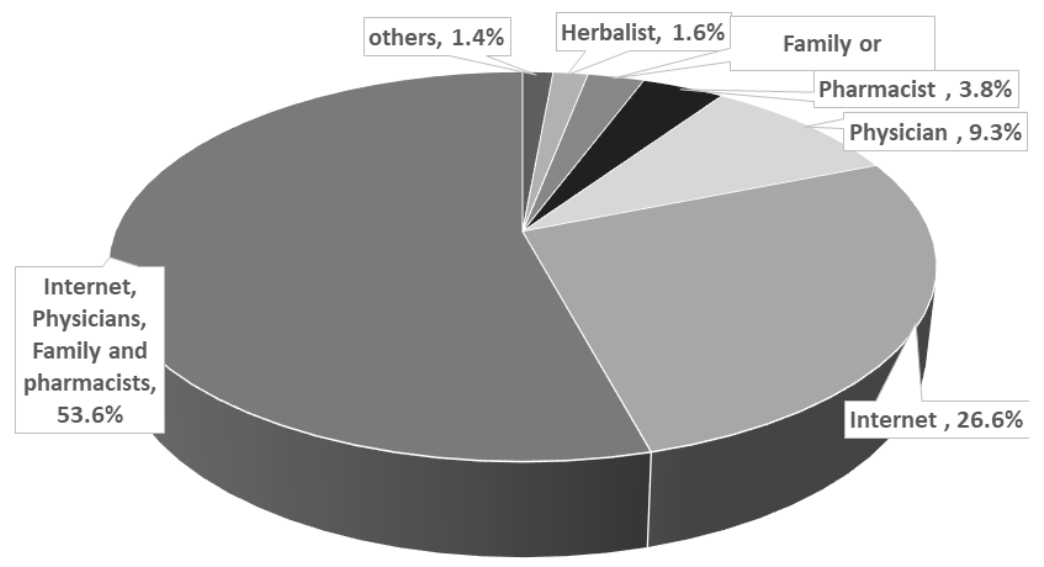

Figure 2. Sources of information about herbs and herbal products among study participants $(\mathrm{N}=$ 789). 